\title{
BMJ Open Longitudinal cohort study of the impact of specialist cancer services for teenagers and young adults on quality of life: outcomes from the BRIGHTLIGHT study
}

Rachel M Taylor (D) , ${ }^{1}$ Lorna A Fern, ${ }^{2}$ Julie Barber, ${ }^{3}$ Javier Alvarez-Galvez (D) , Richard Feltbower, ${ }^{5}$ Sarah Lea, ${ }^{2}$ Ana Martins, ${ }^{2}$ Stephen Morris (1) , ${ }^{6}$ Louise Hooker, ${ }^{7}$ Faith Gibson, ${ }^{8,9}$ Rosalind Raine, ${ }^{10}$ Dan P Stark, ${ }^{11}$ Jeremy Whelan (D) ${ }^{2}$

To cite: Taylor RM, Fern LA, Barber J, et al. Longitudinal cohort study of the impact of specialist cancer services for teenagers and young adults on quality of life: outcomes from the BRIGHTLIGHT study. BMJ Open 2020;10:e038471. doi:10.1136/ bmjopen-2020-038471

- Prepublication history and additional material for this paper is available online. To view these files, please visit the journal online (http://dx.doi.org/10. 1136/bmjopen-2020-038471).

Received 11 March 2020 Revised 07 October 2020 Accepted 16 October 2020

Check for updates

(c) Author(s) (or their employer(s)) 2020. Re-use permitted under CC BY-NC. No commercial re-use. See rights and permissions. Published by BMJ.

For numbered affiliations see end of article.

Correspondence to

Dr Lorna A Fern;

lorna.fern@nhs.net

\section{ABSTRACT}

Objectives In England, healthcare policy advocates specialised age-appropriate services for teenagers and young adults (TYA), those aged 13 to 24 years at diagnosis. Specialist Principal Treatment Centres (PTC) provide enhanced TYA age-specific care, although many still receive care in adult or children's cancer services. We present the first prospective structured analysis of quality of life (QOL) associated with the amount of care received in a TYA-PTC

Design Longitudinal cohort study.

Setting Hospitals delivering inpatient cancer care in England.

Participants 1114 young people aged 13 to 24 years newly diagnosed with cancer.

Intervention Exposure to the TYA-PTC defined as patients receiving NO-TYA-PTC care with those receiving ALL-TYAPTC and SOME-TYA-PTC care.

Primary outcome Quality of life measured at five time points: $6,12,18,24$ and 36 months after diagnosis.

Results Group mean total QOL improved over time for all patients, but for those receiving NO-TYA-PTC was an average of 5.63 points higher $(95 \% \mathrm{Cl} 2.77$ to 8.49$)$ than in young people receiving SOME-TYA-PTC care, and 4.17 points higher $(95 \% \mathrm{Cl} 1.07$ to 7.28$)$ compared with ALL-TYA-PTC care. Differences were greatest 6 months after diagnosis, reduced over time and did not meet the 8-point level that is proposed to be clinically significant. Young people receiving NO-TYA-PTC care were more likely to have been offered a choice of place of care, be older, from more deprived areas, in work and have less severe disease. However, analyses adjusting for confounding factors did not explain the differences between TYA groups.

Conclusions Receipt of some or all care in a TYA-PTC was associated with lower QOL shortly after cancer diagnosis. The NO-TYA-PTC group had higher QOL 3 years after diagnosis, however those receiving all or some care in a TYA-PTC experienced more rapid QOL improvements. Receipt of some care in a TYA-PTC requires further study.

\section{Strengths and limitations of this study}

- We present the first national evaluation of a model of care which aims to improve outcomes for teenagers and young adults with cancer.

- We were able to quantify where young people received care through nationally collated hospital activity data so we could objectively assign young people to a group representing the model of care received.

- Analysis of longitudinal data for 3 years after diagnosis was adjusted for multiple confounding variables, identified from a conceptual model of patient experience, which underpinned data collection in the study.

- The measure quantifying where care was received was based on the assumption that all teenage and young adult Principal Treatment Centres provided equivalent facilities and care.

- The cohort comprises $20 \%$ of young people diagnosed with cancer during the time period, which could impact on the generalisability of the results.

\section{INTRODUCTION}

Cancer in teenagers and young adults (TYA) is uncommon. Despite this, cancer in young people aged 15 to 29 years at diagnosis accounts for an estimated 350000 new incident cases and incidence rates are rising. ${ }^{1}$ Lower survival rates than younger children in several common cancer types ${ }^{2}$ have fuelled many international initiatives aimed to improve outcomes and well-being. ${ }^{34}$ In particular, the need for specialist age-appropriate care and environments are advocated as a critical component of good cancer care for TYA. ${ }^{5-9}$ However, the effect on clinical and patient-reported outcomes associated with age-appropriate care are yet to be described. ${ }^{10}$ 
Distinct cancer service provision for TYA began in the UK in the 1990s. ${ }^{11}$ This was initiated by clinician and patient advocacy, promoting principles which responded to young people's reports of care that frequently lacked support for their priorities of progress towards normal life goals and for care to be delivered alongside others of a similar age and by professionals who understood young people.' . ${ }^{12}$ Specialised services for young people being treated for cancer within the National Health Service (NHS) have been mandated in England since 2005 by the National Institute for Health and Clinical Excellence (NICE) guidance. ${ }^{3}$ The guidance identified that young people's needs may be poorly met in children's and older adult services working in isolation from each other, ${ }^{4}$ and that TYA-specific places of treatment and care may be key to achieving better outcomes for young people with cancer $^{9}$ due in part to the distinct impact of cancer on young people's well-being, such as in the physical, psychosocial and developmental domains. ${ }^{13}$

\section{The delivery of cancer care for TYA in England}

Healthcare in England is in the main, publicly funded through the NHS providing universal comprehensive healthcare to all citizens. 'The service is configured to improve, prevent, diagnose and treat physical and mental health problems with equal regard. ${ }^{, 14}$ Secondary care is delivered in NHS Trusts. Each Trust has its own Chief Executive and leadership team and while they are governed by central NHS legislation, each Trust works as an independent entity. The configuration and delivery of services is therefore unique to each Trust, ensuring that each geographical region in England has access to essential healthcare services. Healthcare delivery by a Trust could be provided in a single hospital providing all the required services or it could be a merger of multiple hospitals in the geographical area each providing specific services (for example: https://www.uclh.nhs.uk/OurServices/OurHospitals/Pages/Home.aspx) ${ }^{15}$

The model of specialised TYA cancer care implemented from 2005 focussed on 13 TYA Principal Treatment Centres (TYA-PTC) in England. These were based in 13 different Trusts, selected to complement the existing services delivered in children's cancer units (aged up to 16 years in the main but local variation would accept older teenagers) and adult cancer services from 18 years onward. The TYA-PTCs were funded to deliver specialist care, which included the same standard of cancer care as the children and adult units but care was enhanced by the addition of age-appropriate environments and multidisciplinary teams experienced in working with TYA. For example, providing education and career support to enable TYA to continue with education and employment at a critical time in their lives; nurse specialists who were skilled at discussing challenging subjects (sex, fertility, drug and alcohol use); space to interact with other TYA with cancer to promote normal development and youth support coordinators who provide youth support and facilitate peer-to-peer activities (see Morgan $e t a l^{7}$ for examples of what is included in a TYA unit).

The location of the TYA-PTCs were chosen based on a number of factors, including existing established service, geographical location and other cancer services available. The guidance directed that TYA aged 16 to 18 years must be treated in a TYA-PTC, while young adults aged 19 to 24 years were to be offered the choice to receive care in a TYA-PTC or a local cancer unit that could provide their cancer treatment and some aspects of ageappropriate care. There was variation in the lower age of admission in TYA-PTCs based on history and availability of other services locally so this resulted in TYA aged 13 to 16 being treated in a children's cancer unit or TYA-PTC and those aged 17 to 24 could be admitted to a TYA-PTC or adult cancer unit. By 2010, about twothirds of those aged 15 to 18 years and one-third of 19 to 24 year olds were believed to have contact with a TYAPTC. ${ }^{16}$ Place of care was therefore directed by clinicians based on cancer type and geographical location. While there is an international mobilisation to implement specialist TYA services, ${ }^{12}$ including in other European countries, Australasia and North America, the impact of such services on clinical outcomes has not been robustly evaluated.

The BRIGHTLIGHT study is a prospective evaluation of the benefits of specialist TYA services in the English NHS. In order to capture the complexity of the delivery of TYA cancer care, it comprised an evaluation from the perspective of the environment of care ${ }^{17}$ the workforce delivering care ${ }^{18}$ and young people receiving care. BRIGHTLIGHT was developed with extensive input from young people as well as health professionals ${ }^{6919}$ and based on consultation with young people. ${ }^{20}$ This included input into the selection of the primary outcome: quality of life (QOL).

Quality of life is defined as '...subjective, multidimensional and dynamic. It is unique to each individual and includes aspects of physical, psychological and social function. It is dependent not only on the stage of development but also the illness trajectory. This involves the achievement of goals and aspirations and the constraints imposed through ill health and treatment'. ${ }^{21}$ Measurement of QOL uses the patient's own report to evaluate the spectrum of impact of illness on them and has become an increasingly valued healthcare outcome. Previous reports of young people's QOL after a cancer diagnosis have shown this to be significantly lower than normative population data. ${ }^{22}$ Longitudinal assessment has indicated QOL improved in the first year after diagnosis but there was no significant improvement in the second year. ${ }^{23}$ No evaluation beyond the second year has been reported and while studies have investigated predictors of QOL there has been no evaluation of the impact of different models of delivery of care on QOL. We examined QOL at 6, 12, 18, 24 and 36 months after diagnosis in relation to the amount of care young people received in a TYA-PTC. 


\section{METHODS}

\section{Study design}

The BRIGHTLIGHT study is a mixed methods programme of research. Results from an embedded longitudinal cohort study, obtaining data from young people through a bespoke survey, ${ }^{24}$ are reported here. The survey was administered at five time points during the first 3 years after diagnosis $(6,12,18,24$ and 36 months, waves 1 to 5 respectively). A scale was developed, previously described in detail, ${ }^{25}$ using Hospital Episode Statistics (HES) inpatient data to measure episodes of care in different NHS Trusts and then used to assign young people to one of three categories of TYA care dependent on how much inpatient HES activity recorded in the first 12 months after diagnosis was delivered in a TYA-PTC: no care in a TYA-PTC, that is, all care was delivered in a children's or adult cancer unit (NO-TYA-PTC), some care delivered in a TYA-PTC with additional care in a children's or adult cancer unit (SOME-TYA-PTC) or all care delivered in a TYA-PTC (ALL-TYA-PTG).

\section{Patient and public involvement}

BRIGHTLIGHT has been developed with young people from the point of inception and our Young Advisory Panel (YAP) have been involved in the management, implementation and dissemination of the study. This has been reported in detail previously ${ }^{2026-29}$ but in summary, BRIGHTLIGHT was developed based on consultation with young people attending a patient conference in 2008: place of care was identified as the third priority for future research. Young people worked with the research team to conduct the research informing the National Institute for Health Research grant application ${ }^{6}$ including representation as a co-applicant. The YAP have advised on changes to recruitment, ${ }^{28}$ helped develop the retention strategy, ${ }^{26}$ informed additional studies ${ }^{29}$ and are involved in dissemination. ${ }^{30}$

\section{Participants and setting}

BRIGHTLIGHT was open to recruitment between October 2012 and April 2015 in 109 English NHS hospitals of which 97 recruited at least one young person. Eligibility was defined as aged 13 to 24 years at the time of diagnosis, newly diagnosed with cancer (ICD-10 (International Classification of Diseases, 10th Revision) codes C00-C97) and recruited within 4 months of diagnosis. There was no eligibility exclusion for a language or sensory impairment affecting communication. The following groups were excluded: those serving a custodial sentence; not anticipated to be alive at the first point of data collection (6 months after diagnosis); or incapable of completing a survey. Details of the recruitment process are reported elsewhere. ${ }^{25} 31$ Young people gave written consent and parental consent was also obtained for those less than 16 years. Checks were made through the Demographic Batch Service at NHS digital before each wave of data collection to ensure young people were alive and to obtain their most recent address.
The sample size calculation was based on a comparison between the three categoriesof TYA care $^{25}$ for the primary outcome of PedsQL (Paediatric Quality of Life Questionnaire) total score, ${ }^{32}$ measured at five time points over the 3-year follow-up. Previously reported PedsQL data for childhood patients with cancer suggested a SD for this score of $16 .^{33}$ To detect a difference in scores of eight units with $80 \%$ power $^{34}$ required a sample of 200 young people. This calculation assumed a significance level of 0.01 (two-sided) to allow for multiple comparisons between three categories of TYA care and assumed an average of three repeated measurements per patient (intracluster correlation 0.3 suggested as a maximum for similar patient outcomes ${ }^{35}$ ). The calculation allowed for adjustment for confounding factors using a variance inflation factor with a correlation of $0.5 .^{36}$ To ensure adequate power to examine the effect of TYA care on QOL within subgroups of age at diagnosis (13 to 18 and 19 to 24 years) and type of tumour (haematological and solid tumour groups), the minimum required sample size was raised to $800(80 \%$ power $)$.

\section{Data collection}

Data were collected from three sources: young people's self-report, patient clinical records and NHS and Public Health England databases. Details of these data sources are reported elsewhere. ${ }^{25}$ Data presented here are responses to the BRIGHTLIGHT Survey, a bespoke survey containing five validated questionnaires and 169 descriptive questions related to post diagnosis experience. The survey was administered through face-to-face interviews in young people's homes by an independent research company at the first time point and either online or telephone interview at subsequent waves of data collection. ${ }^{24}$

This paper reports data for the primary outcome, QOL, which was measured using the PedsQL. ${ }^{32}$ At the time of study development this was the only measure of QOL validated for TYA ${ }^{37}$ It contains 23 items rated using a 5-point Likert Scale (never, almost never, sometimes, often and almost always). Responses are presented as four domain scores (physical, emotional, social and work/studies functioning), two summary scores (physical and psychosocial function) and a total score. Domain, summary and total scores range from 0 to 100 , with 100 representing the best possible QOL.

\section{Analysis}

Analysis was carried out following a predefined statistical analysis plan using Stata V.15. A mixed effects model was used to investigate the relationship between the categories of TYA care and the PedsQL total score, allowing for repeated measurements taken over the 3 years since diagnosis. The model was adjusted for confounding factors identified based on the conceptual model underpinning the BRIGHTLIGHT Survey ${ }^{64}$ and using a causal diagram in the form of a Directed Acyclic Graph (DAG) (DAGitty software www.dagitty.net; online supplemental figure 1). Factors adjusted for were age at diagnosis, type of 
cancer (leukaemia, lymphoma, brain and central nervous system, bone tumours, sarcoma, germ cell, melanoma, carcinomas and other), socioeconomic status (Index of Multiple Deprivation ${ }^{38}$ quintile), severity of cancer (least, intermediate and $\operatorname{most}^{25}$ ), ethnicity (white and other), choice offered about where to receive treatment (yes/no), presence of any long-term condition prior to cancer (yes/ no), days from first symptom to diagnosis and number of general practitioner visits before diagnosis. Geographical location (specified as 12 cities, derived from the TYA-PTC and their network of hospitals) was included in the model as a random effect. Models were extended to include interaction terms to investigate predefined subgroup effects by age at diagnosis (both as a continuous factor and using categories of 13 to 18 and 19 to 24 years) and tumour type (haematological and solid tumours). An interaction with time since diagnosis was also examined to investigate whether the relationship betweencategory of TYA care and QOL changed over time. Similar models were fitted for the PedsQL domain scores for physical, social, emotional and work/school/college functioning, and the psychosocial summary score.

The extent and patterns of missing QOL data over time were examined using summary statistics and profile plots. As there is no provision in the scoring of PedsQL to directly account for death, our main analysis did not distinguish between data 'missing' following death and that missing for other reasons. With the possibility of informative missing data due to deaths, a sensitivity analysis was carried where joint mixed-effect models for the longitudinal QOL scores and time until death were fitted to account for the correlation between the QOL and survival outcomes. ${ }^{39}$ The QOL estimates for the effect of category of TYA care were then compared with those obtained from previously fitted mixed models.

\section{RESULTS}

A total of 1126 young people were recruited. BRIGHTLIGHT survey data at wave 1 were available for 830 $(75 \%)$ participants and details of numbers at each wave are summarised in figure 1. Characteristics of those who did and did not complete wave 1 were similar, except for a higher proportion of non-white participants not completing wave 1 ( $12 \%$ vs $19 \%, \mathrm{p}=0.004) .{ }^{25}$ Forty-eight participants could not be assigned acategory of TYAPTC care as there were no linked HES inpatient records available. Data from 782 young people were therefore included. There were fewer young people receiving ALLTYA-PTC care $(\mathrm{n}=193 ; 25 \%)$ in comparison to SOMETYA-PTC $(\mathrm{n}=312 ; 40 \%)$ and NO-TYA-PTC $(\mathrm{n}=277 ; 35 \%)$. Demographic characteristics and summary of variables adjusted for in the analysis are shown in table 1. Young people who received NO-TYA-PTC care were slightly older, more likely to be working full-time/part-time, had less severe disease, had a better prognosis and were more likely to have been given a choice in their place of care.

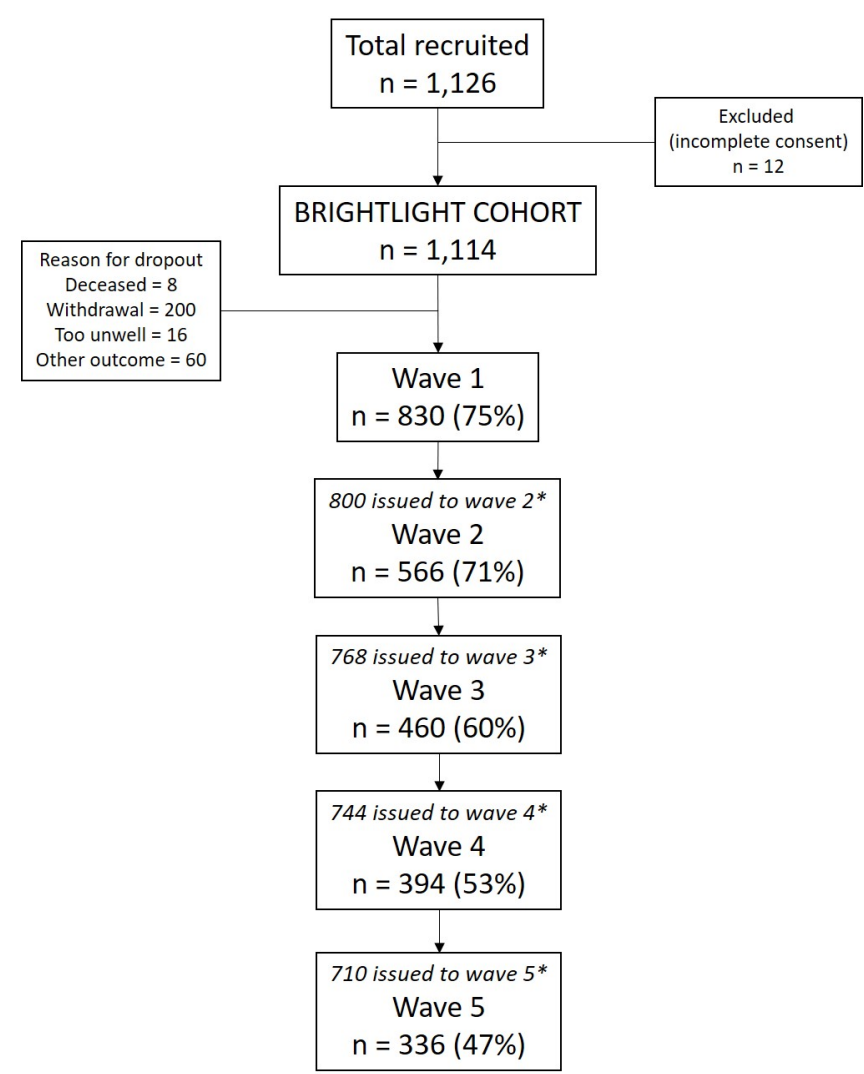

Figure 1 Participation at each wave of data collection. *Drop-outs between waves due to death, permanent opt-out or wave opt-out. Wave opt-outs prior to being issued were not permanent opt-outs; participants could opt-out of a single wave but participate in subsequent waves; these cases were not removed from the cohort permanently.

Young people who had ALL-TYA-PTC care were more likely to come from less deprived areas.

Longitudinal changes in the average QOL total score according to the category of care are shown in figure 2 (summary by wave is given in online supplemental table 1). Across all time periods this illustrates lower QOL score for those in the SOME-TYA-PTC and ALL-TYA-PTC groups compared with the NO-TYA-PTC group. Differences between the category of care appeared to diminish steadily over time. The negative coefficients for categories of care from the model indicate that across the 3-year period of the study QOL scores were on average higher for those with NO-TYA-PTC care compared with SOME-TYA-PTC and ALL-TYA-PTC with a larger difference compared with the SOME-TYA-PTC category (table 2). The differences were statistically significant but fell inside the threshold of eight points proposed to be clinically significant. ${ }^{32}$ There was no evidence that the relationship between QOL and category of care was different for the combined group of leukaemia/ lymphomas compared with other cancers. Also, there was no evidence of a difference by age at diagnosis (figure 3 ). There was evidence that the QOL/category of care relationship changed over time such that differences between the groups diminished, that is, slopes for SOME-TYA-PTC and ALL-TYA-PTC over time are steeper than for NO-TYA-PTC, 
Table 1 Participant characteristics according to category of care at wave 1

\section{Characteristic}

Category of TYA care at $\mathbf{1 2}$ months from diagnosis

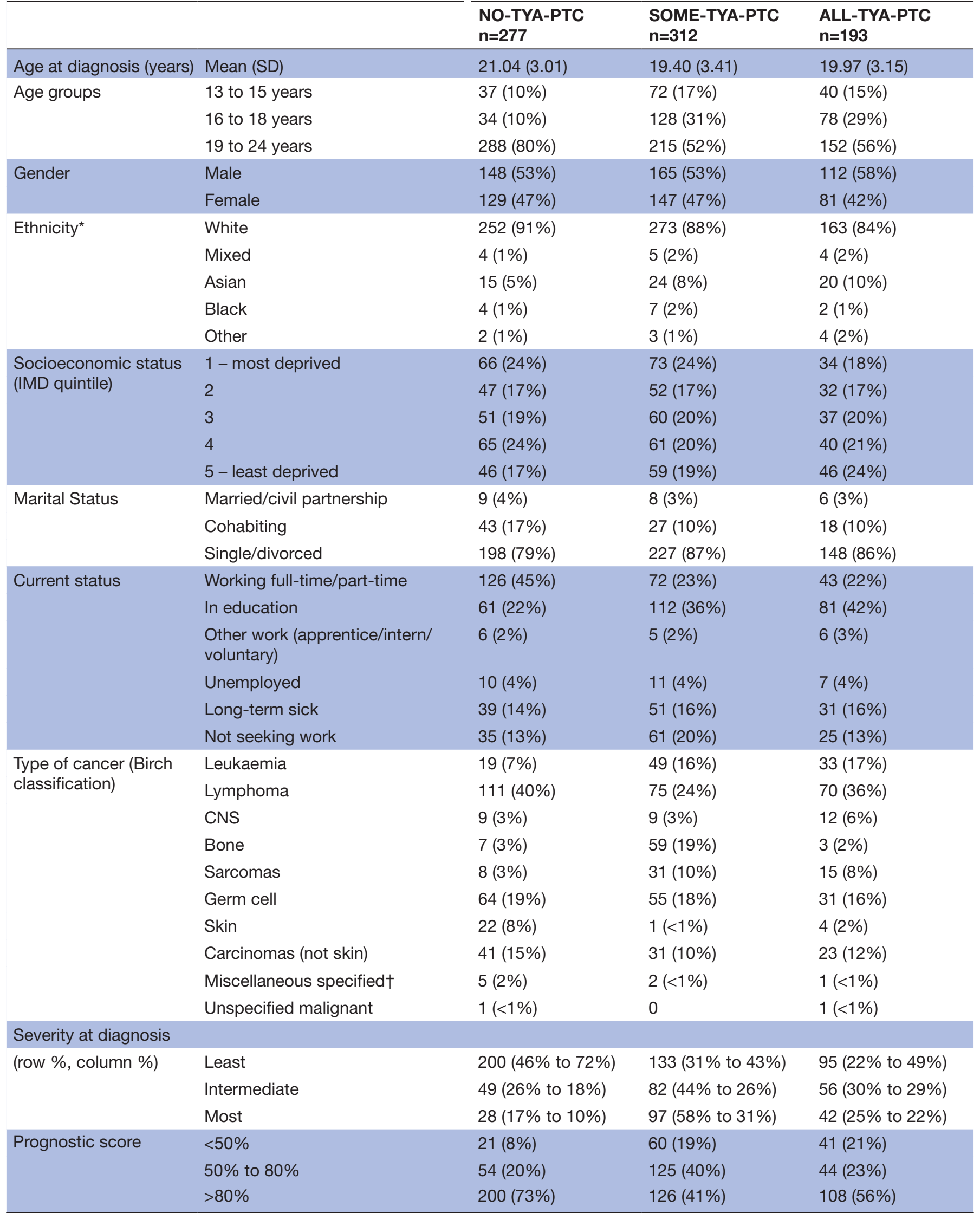

Continued 
Table 1 Continued

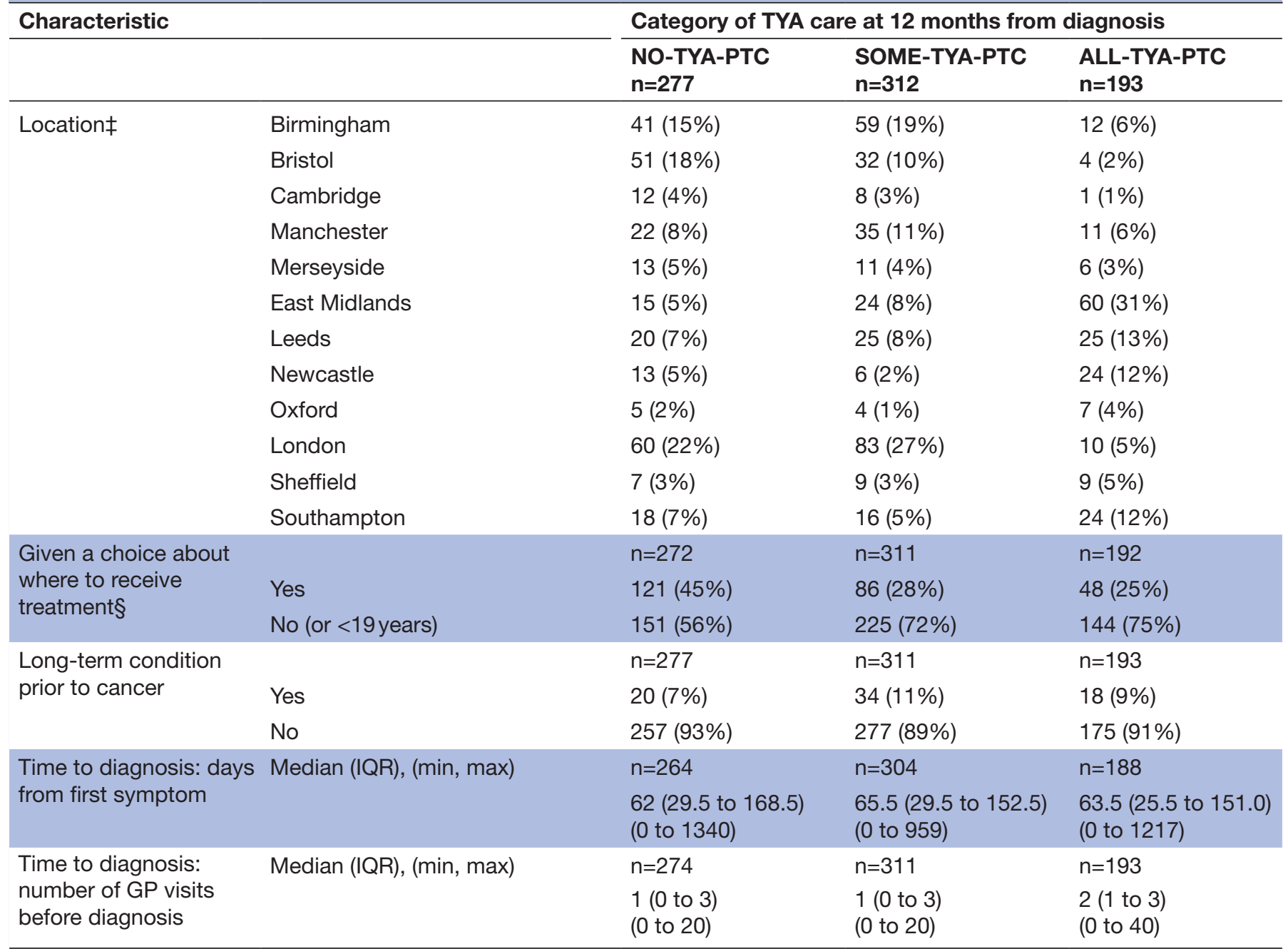

*Wave 1 data was used with missing values completed using available Public Health England data.

†Includes 4 'unclassified' - treated in cancer unit but did not have cancer

$\ddagger$ Those $<19$ years at diagnosis were assumed not to have been given a choice

§Includes the TYA-PTC and hospitals linked to the multidisciplinary team at the TYA-PTC; where available based on hospital of diagnosis, for

77 cases based on recruiting hospital

CNS, central nervous system; GP, general practitioner; IMD, index of multiple deprivation; TYA, teenagers and young adults; TYA-PTC, TYA principal treatment centres.

therefore rates of improvement of QOL were superior in the ALL-TYA-PTC and SOME-TYA-PTC (table 3), mirroring what was seen in figure 2.

Analysis of the physical functioning and work/school/ college functioning domain scores showed a similar pattern to that seen for the total QOL score. The SOMETYA-PTC group had a mean difference in physical functioning of -8.28 compared with the NO-TYA-PTC group, which was statistically significant and above the threshold for clinical significance. For the emotional functioning domain however, difference between groups were less marked and notably the average difference in scores between the SOME-TYA-PTC and NO-TYA-PTC was reduced. Difference between groups in terms of social functioning and the psychosocial summary scores were small and not statistically significant (figures 4-8, table 2).
Results from post-hoc analysis excluding patients with bone tumours were similar to those for all cancers combined (see online supplemental table 2). A subgroup investigation of patients with lymphoma, the biggest diagnostic category, showed these were not significantly different from those for the group of other cancer types (see online supplemental table 3 ). Finally, sensitivity analyses using joint modelling for survival alongside longitudinal QOL did not alter the conclusion based on the main results (see online supplemental table 4).

\section{DISCUSSION}

Our study is the first reported national longitudinal evaluation of specialist cancer services, more specifically TYAPTCs and their networks of care, as they were originally 


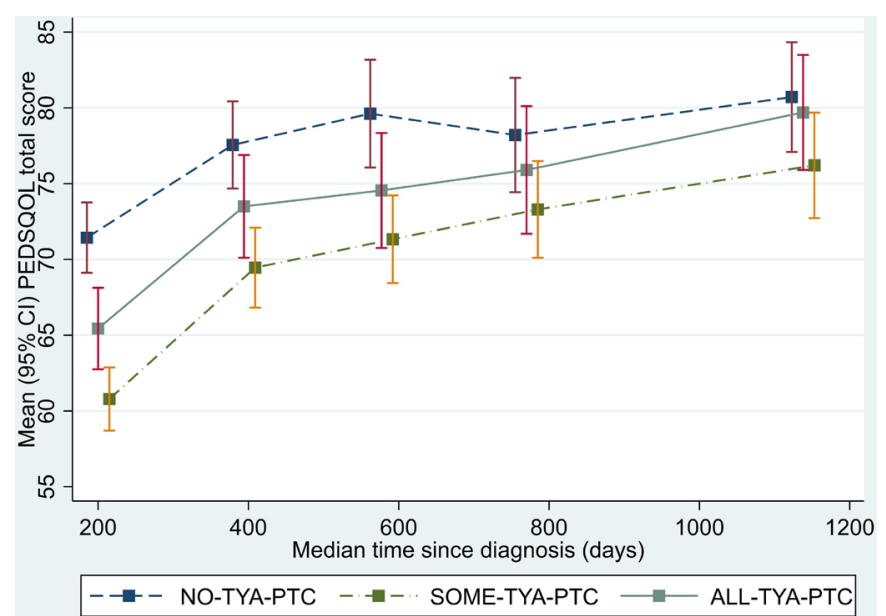

Figure 2 Mean PedsQL (Paediatric Quality of Life Questionnaire) total score over time since diagnosis (with 95\% Cls). Graph based on data collected in Waves 1 to 5 , plotted against median time since diagnosis. TYA, teenagers and young adults; TYA-PTC, TYA principal treatment centres.

defined in $2005 .{ }^{3}$ We recorded patient outcomes to determine the influence of place of care, and after consultation with patients and professionals, selected QOL as the primary parameter of interest. Using routinely collected hospital admission data to identify time spent in the

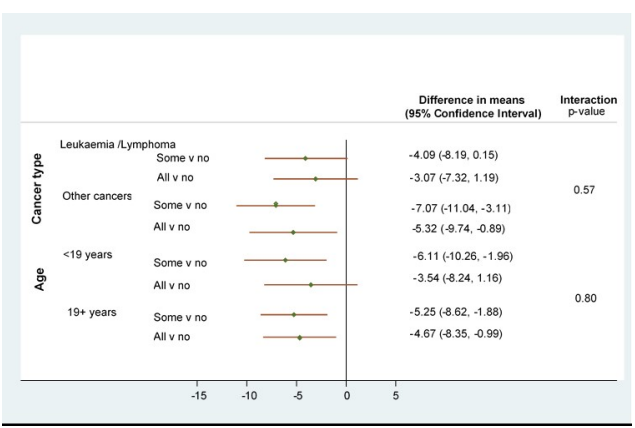

Figure 3 Subgroup investigations for cancer type (leukaemia/lymphoma $\mathrm{v}$ other) and age group $(<19 \mathrm{v}$ 19+): Results from adjusted* random effects models with interaction terms $(n=733)$. *Model with a random effect for city and adjustment for time since diagnosis, age at diagnosis, type of cancer, socioeconomic status, severity of cancer, ethnicity, choice about where to receive treatment, long-term condition prior to cancer, days from first symptom to diagnosis, number of general practitioner visits before diagnosis. v, versus.

TYA-PTC, we categorised patients by care received in the TYA-PTC (all, none or some). We found that there was a statistically significant difference between these groups with those in the NO-TYA-PTC group having superior QOL compared with those in the ALL-TYA-PTC group,

Table 2 Mixed model investigating the relationship between categories of TYA care received during the first 12 months from diagnosis and total quality of life and domain scores over 3 years $(n=733)$

\begin{tabular}{|c|c|c|c|c|}
\hline & & Difference in means & $95 \% \mathrm{Cl}$ & $\mathbf{P}$ value \\
\hline \multicolumn{5}{|l|}{ Total quality of life score $(n=733)$} \\
\hline TYA care category (v NO-TYA-PTC) & SOME-TYA-PTC & -5.63 & -8.49 to -2.77 & $\mathrm{p}=0.0005$ \\
\hline \multicolumn{5}{|l|}{ Physical functioning ( $n=733)$} \\
\hline TYA care category (v NO-TYA-PTC) & SOME-TYA-PTC & -8.28 & -11.95 to -4.61 & $\mathrm{p}=0.0001$ \\
\hline \multicolumn{5}{|l|}{ Emotional functioning $(n=733)$} \\
\hline \multirow[t]{2}{*}{ TYA care category (v NO-TYA-PTC) } & SOME-TYA-PTC & -4.29 & -7.79 to -0.80 & $\mathrm{p}=0.015$ \\
\hline & ALL-TYA-PTC & -5.43 & -9.29 to -1.57 & \\
\hline \multicolumn{5}{|l|}{ Social functioning $(n=733)$} \\
\hline TYA care category (v NO-TYA-PTC) & SOME-TYA-PTC & -2.96 & -5.77 to -0.16 & $p=0.099$ \\
\hline \multirow[t]{2}{*}{ TYA care category (v NO-TYA-PTC) } & SOME-TYA-PTC & -6.87 & -10.45 to -3.30 & $p=0.0007$ \\
\hline & ALL-TYA-PTC & -4.67 & -8.47 to -0.87 & \\
\hline \multicolumn{5}{|l|}{ Psychosocial summary score $(n=600)$} \\
\hline \multirow[t]{2}{*}{ TYA care category (v NO-TYA-PTC) } & SOME-TYA-PTC & -2.51 & -5.71 to 0.70 & $\mathrm{p}=0.074$ \\
\hline & ALL-TYA-PTC & -3.96 & -7.44 to -0.48 & \\
\hline
\end{tabular}

${ }^{*}$ Results from a three category of care model for repeated measurements of quality of life over time since diagnosis, with a random effect for geography and adjustment for time since diagnosis, age at diagnosis, type of cancer, socioeconomic status, severity of cancer, ethnicity, choice about where to receive treatment, long-term condition prior to cancer, days from first symptom to diagnosis, number of general practitioner visits before diagnosis. Missing data for 96 due to missing TYA category and missing data in other covariates. †Likelihood ratio test.

TYA, teenagers and young adults; TYA-PTC, TYA principal treatment centres. 
Table 3 Investigation of changes in QOL score over time: results from adjusted mixed effects models with interaction term (time months x TYA-PTC category). Coefficients for time describe the linear increase in QOL score per month within each TYA care category $(n=733)$

\begin{tabular}{llll}
\hline TYA care category & Coefficient for time (per month) & $\mathbf{9 5 \%} \mathbf{C l}$ & P value from interaction \\
\hline NO-TYA-PTC & 0.26 & 0.18 to 0.34 & 0.004 \\
SOME-TYA-PTC & 0.45 & 0.37 to 0.53 & \\
ALL-TYA-PTC & 0.37 & 0.27 to 0.46 & \\
\hline
\end{tabular}

Model with a random effect for city and adjustment for time since diagnosis, age at diagnosis, type of cancer, socioeconomic status, severity of cancer, ethnicity, choice about where to receive treatment, long-term condition prior to cancer, days from first symptom to diagnosis, number of general practitioner visits before diagnosis.

QOL, quality of life; TYA, teenagers and young adults; TYA-PTC, TYA principal treatment centres.

while those in the SOME-TYA-PTC group reported lowest QOL. Quality of life improved over time in all three groups, but rates of improvement were significantly better for SOME-TYA-PTC and ALL-TYA-PTC.

We predicted that sociodemographic or disease factors might explain some of the differences between groups and adjusted the analyses for these confounding variables. Despite extensive analysis we were unable to identify other factors to account for these differences. Like other reports of young people's QOL after a cancer diagnosis, ${ }^{22}$ we found this to be low, irrespective of where young people were treated. We found that young people who did not access a TYA-PTC had better QOL in comparison to those who had all or some of their care in a TYA-PTC. However, while this was statistically significant, the mean difference between the NO-TYA-PTC and ALL-TYA-PTC groups was not at a level proposed as clinically significant (eight-point difference ${ }^{33}$ ). Nevertheless, it is important to consider reasons for lower QOL when young people experience multiple types of place of care and the determinants of place of care. Based on work in other settings where care is delivered on multiple sites ${ }^{40}$ we surmise that

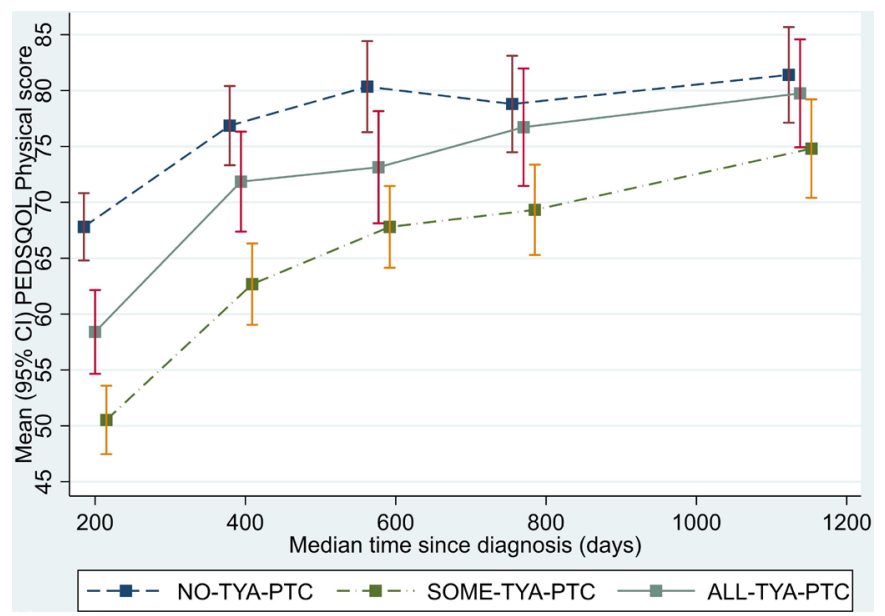

Figure 4 Mean PedsQL (Paediatric Quality of Life Questionnaire) physical functioning domain scores over time since diagnosis (with 95\% Cls). (Graphs based on data collected in Waves 1 to 5, plotted against median time since diagnosis).TYA, teenagers and young adults; TYA-PTC, TYA principal treatment centres. this may result from limited coordination of care, perhaps including inadequate communication with and between professionals. Having to repeat conversations and explanations of their cancer diagnosis and treatment details is frequently reported as burdensome to young people. ${ }^{6}$ A greater understanding of the determinants of place of care for young people and the factors which influence a sense of care coordination deserve further exploration.

It is interesting that young people who had no access to the TYA-PTC rated their QOL the highest. This could reflect young people rating themselves by comparison with the other people they could see being treated for cancer outside of a TYA-PTC, including older adults. It could also be that young people chose to receive care locally rather than travel to the TYA-PTC so they could keep their links to their 'normal' life, which is supported by the domain level analysis where they also rated their work/school/college functioning higher. Alternatively, the strong emphasis placed on the unique issues faced by TYA with cancer by members of the TYA MDT staff may have heightened patients' awareness of these problems in comparison to the NO-TYA-PTC group, and consequently

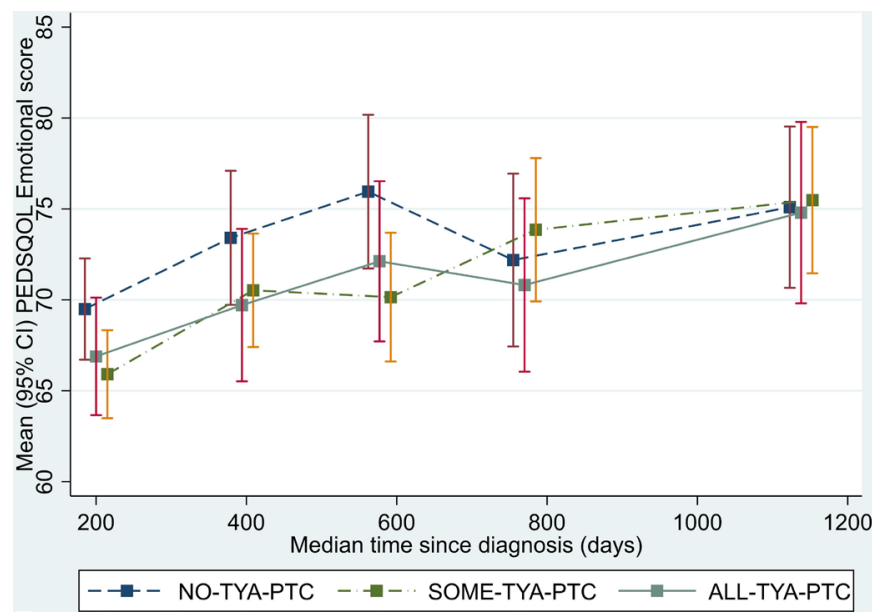

Figure 5 Mean PedsQL (Paediatric Quality of Life Questionnaire) emotional functioning domain scores over time since diagnosis (with 95\% Cls). (Graphs based on data collected in Waves 1 to 5 , plotted against median time since diagnosis). TYA, teenagers and young adults; TYA-PTC, TYA principal treatment centres. 


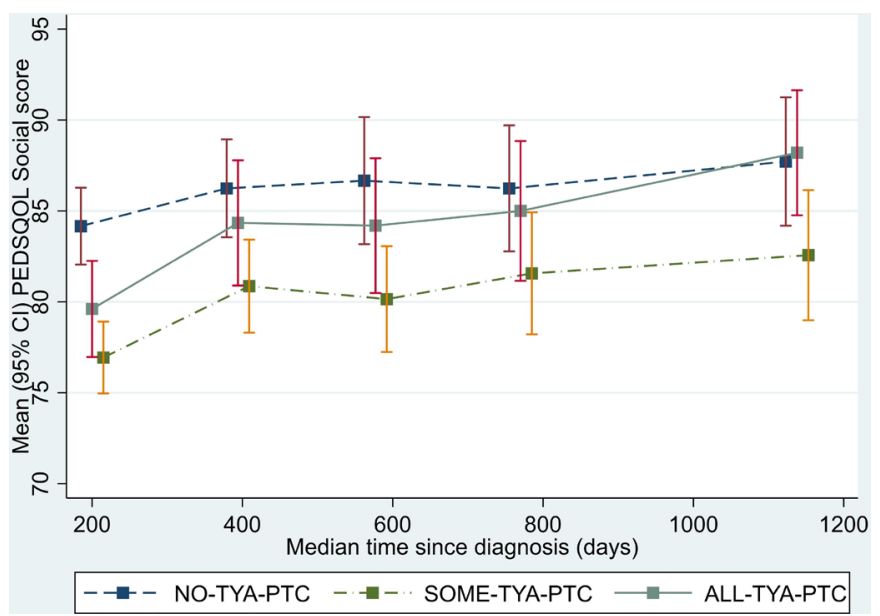

Figure 6 Mean PedsQL (Paediatric Quality of Life Questionnaire) social functioning domain scores over time since diagnosis (with 95\% Cls). (Graphs based on data collected in Waves 1 to 5, plotted against median time since diagnosis). TYA, teenagers and young adults; TYA-PTC, TYA principal treatment centres.

they lowered their perception of their QOL while the NO-TYA-PTC group remained comparatively unaware of such concerns. Future work could focus on the influence of being given a choice in the place where young people receive care, and the factors that young people consider to be important when making this decision.

The longest follow-up for longitudinal assessment of QOL previously reported to 2 years after diagnosis, ${ }^{23}$ which showed no improvement after the first year. However, we found that there was a gradual improvement in QOL over 3 years, which was more rapid when young people received all their care in a TYA-PTC. The philosophy of TYA cancer care includes the delivery of care to support young people to achieve their long-term personal outcomes (education, employment and relationships),

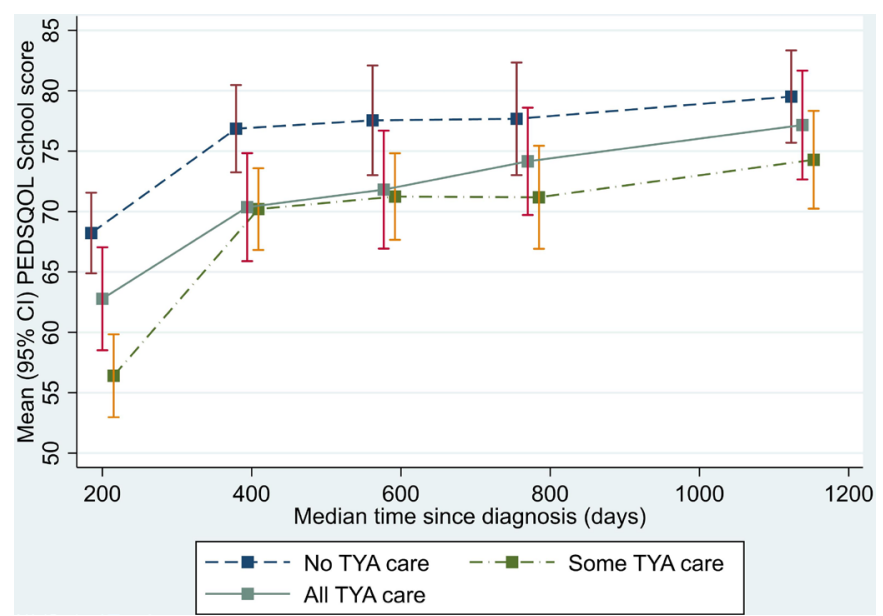

Figure 7 Mean PedsQL (Paediatric Quality of Life Questionnaire) work/school/college functioning domain scores over time since diagnosis (with 95\% Cls). (Graphs based on data collected in Waves 1 to 5 , plotted against median time since diagnosis). TYA, teenagers and young adults; TYA-PTC, TYA principal treatment centres.

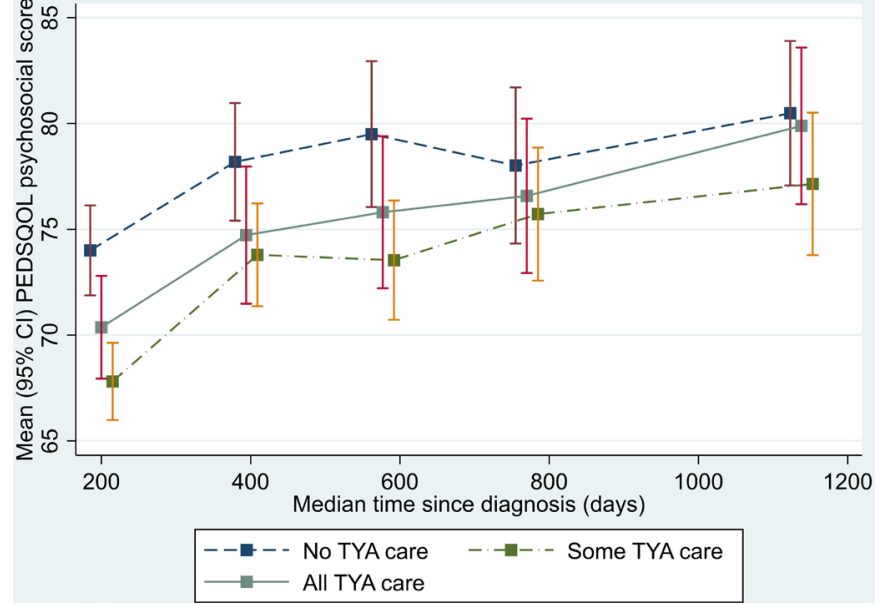

Figure 8 Mean PedsQL (Paediatric Quality of Life Questionnaire) psychosocial summary score scores over time since diagnosis (with 95\% Cls). (Graphs based on data collected in Waves 1 to 5, plotted against median time since diagnosis).

the benefits of care provided by the TYA-PTC may therefore not be realised in the short-term. It would be beneficial for us to understand whether this improvement continued into long-term survivorship, especially the influence of QOL reaching and sustaining goals such as employment.

There are several limitations to this study including how specialist care was defined and measured. This was based on the location of the 13 NHS Trusts in England commissioned as TYA-PTCs but not the specific hospitals within these Trusts where the specialist TYA services were based. For example, a Trust which included multiple hospitals could only have specialist TYA services in one therefore a young person receiving care in one of the other hospitals was assumed to have had access to specialist TYA services, that is, they were assigned to the ALL-TYA-PTC group rather than NO-TYA-PTC. Furthermore, using the Trust commissioned as a TYA-PTC does not capture the details of the TYA-specific care available or delivered and assumes that this is equal in all. We know there was wide variation in the delivery of care through the duration of the study. ${ }^{41}$ However, at the time of study inception HES was the sole data source available that would allow an objective measurement of place of care. In complementary work, the key elements of specialist age-appropriate care for TYA have been described. ${ }^{17}$ This would provide an alternative categorisation against which to measure patient and clinical outcomes.

The cohort represented approximately a fifth of the total cancer population diagnosed between July 2012 and December 2014 as ascertained through the National Cancer Registration and Analysis Service, and there were differences in cancer types between the cohort and those not recruited, ${ }^{25}$ which could impact on the generalisability of the results. For example, the cohort included a higher proportion of young people with germ cell tumours and lymphoma, but a lower proportion of carcinoma and 
skin cancers. We used a single mode of survey administration at wave 1 , and multiple modes at waves 2 to 5 . While this may have introduced a social desirability bias (noted to be more so for telephone interviews than webbased surveys ${ }^{42}$ ), this was to increase the response rate as work during the feasibility study for BRIGHTLIGHT indicated no one method was acceptable to all young people. Finally, we used a measure of QOL that was validated across the age 13 to 24 years, ${ }^{37}$ but this may not reflect the issues that were most relevant to young people with cancer in the UK (having been developed in the USA in a non-cancer population). This is supported by comments made in the cognitive interviews undertaken when the survey was being developed; young people did not agree with the wording of the school functioning domain, so this was changed to work/school/college. ${ }^{24}$ However, young people who were not in education, employment or training would not be able to answer these questions. Future work is required to develop TYA-specific QOL measures that reflect issues specific to this population.

Despite these limitations this is the first systematic prospective evaluation of specialist services for young people with cancer. We have found that TYA cancer care as commissioned in 2010 resulted in young people's QOL gradually improving 3 years after diagnosis and improving more rapidly from a lower baseline if young people's treatment involved a TYA-PTC. NHS care pathways may result in care in specialist centres for young people with the poorest initial QOL, and local care for those with the least poor initial QOL, risk stratifying the patients appropriately. Young people who receive some care in both a children's or adult cancer unit and at a TYA-PTC also have an improvement in QOL, but the rate of improvement was less and QOL remained lower than for young people treated in a single type of organisation. The factors influencing place of care and the differences in QOL and survival remain unclear. A model of 'joint care', increasing the emphasis and investment in communication between TYA-PTCs and other Trusts designated to deliver elements of TYA cancer care, is currently proposed by the NHS in England. The influence of such changes in care provision should be examined prospectively in future to identify if QOL of young people with cancer is improved wherever care is received.

\section{Author affiliations}

${ }^{1}$ Centre for Nurse, Midwife and AHP Led Research (CNMAR), University College London Hospitals NHS Foundation Trust, London, UK

${ }^{2}$ Cancer Division, University College London Hospitals NHS Foundation Trust, London, UK

${ }^{3}$ Department of Statistical Science, University College, London, UK

${ }^{4}$ Department of Biomedicine, Biotechnology and Public Health, University of Cadiz, Cadiz, Spain

${ }^{5}$ School of Medicine, University of Leeds, Leeds, UK

${ }^{6}$ Primary Care Unit, Department of Public Health and Primary Care, University of Cambridge, Cambridge, UK

${ }^{7}$ Wessex Teenage and Young Adult Cancer Service, University Hospital Southampton, Southampton, UK

${ }^{8}$ School of Health Sciences, Faculty of Health and Medical Sciences, University of Surrey, Guildford, UK
${ }^{9}$ Centre for Outcomes and Experience Research in Children's Health, IIIness and Disability (ORCHID), Great Ormond Street Hospital for Children NHS Foundation Trust, London, UK

${ }^{10}$ Department of Applied Health Research, University College London, London, UK

${ }^{11}$ Leeds Insitute of Molecular Medicine, University of Leeds, Leeds, UK

\section{Twitter Lorna A Fern @LornaAFern}

Acknowledgements We would like to thank the members of our Young Advisory Panel (Zeena Beale, Ciaran Fenton, Emily Freemantle, Jaasjan Guvindia, Laura Haddard, Steph Hammersley, Joshua Lerner, Tanya Loughlin, Jason, Sin Jin Loo, Jennifer Miller, Maria Onasanya, Arif Nasir, Steph Still, Poppy Richards, Amy Riley, Freya Voss, JJ Wheeler, Max Willliamson, Antonia Young), the 1114 young people who consented to participate in BRIGHTLIGHT, healthcare professionals who approached and consented young people and ex-members of the team who have contributed to study management (Catherine 0'Hara, Anita Solanki, Natasha Aslam, Zuwena Fox). We would like to dedicate this manuscript to the memory of Mr Stephen Sutton, Mr Mathew Cooke, Ms Lara Veitch and Ms Amy Lang all YAP members who were instrumental to study set, design and management. All of whom died from the their cancer during the study. Both of whom died from their cancer during the study. We would also like to thank the following for all their support with recruitment to BRIGHTLIGHT: the National Cancer Research Institute, especially Dr Eileen Loucaides and the Secretariat; Matt Seymour, Matt Cooper and Karen Poole at the former National Cancer Research Network; Maria Khan and Sabrina Sandhu (North West Knowledge Intelligence Team); TYAC; Teenage Cancer Trust; CLIC Sargent; Ipsos MORI; Quality Health and the research teams at 109 NHS Trusts in England who opened BRIGHTLIGHT to recruitment. Principal Investigators agreeing to be acknowledged for their contribution to BRIGHTLIGHT recruitment: Claire Hemmaway, Barking, Havering and Redbridge Hospitals NHS Trust; Anita Amadi, Barnet and Chase Farm Hospitals NHS Trust; Keith Elliott, Barnsley Hospital NHS Foundation Trust; Leanne Smith, Blackpool, Fylde and Wyre Hospitals NHS Trust; Shirley Cocks, Bolton NHS Foundation Trust; Victoria Drew, Bradford Teaching Hospitals NHS Foundation Trust; Elizabeth Pask, Central Manchester University Hospitals NHS Foundation Trust; Anne Littley, Central Manchester University Hospitals NHS Foundation Trust; Mark Bower, Chelsea and Westminster Hospital NHS Trust; Scott Marshall, City Hospitals Sunderland NHS Foundation Trust; Lorna Dewar, Colchester Hospital University NHS Trust; Nnenna Osuji, Croydon Health Services NHS Trust; David Allotey, Derby Hospitals NHS Foundation Trust; Karen Jewers, East Lancashire Hospitals NHS Trust; Asha Johny, Gloucestershire Hospitals NHS Foundation Trust; Nicola Knightly, Great Western Hospitals NHS Foundation Trust; Robert Carr, Guy's and St Thomas' Hospital NHS Foundation Trust; Alison Milne, Hampshire Hospitals NHS Foundation Trust; Claire Hall, Harrogate and District NHS Foundation Trust; James Bailey, Hull and East Yorkshire Hospitals NHS Trust; Christine Garlick, Ipswich Hospital NHS Foundation Trust; Alison Brown, Isle of Wight Healthcare NHS Trust; Carolyn Hatch, Lancashire Teaching Hospitals NHS Foundation Trust; Vivienne E Andrews, Medway NHS Foundation Trust; Sara Greig, Milton Keynes Hospital NHS Foundation Trust; Jennifer Wimperis, Norfolk and Norwich University Hospital NHS Trust; Suriya Kirkpatrick, North Bristol NHS Trust; Jonathan Nicoll, North Cumbria University Hospitals NHS Trust; Ivo Hennig, Nottingham University Hospitals NHS Trust; Karen Sherbourne, Oxford Radcliffe Hospital NHS Trust; Clare Turner, Plymouth Hospitals NHS Trust; Claire Palles-Clark, Royal Surrey County Hospital NHS Trust; Christine Cox, Royal United Hospital Bath NHS Trust; Yeng Ang, Salford Royal NHS Foundation Trust; Jonathan Cullis, Salisbury NHS Foundation Trust; Daniel Yeomanson, Sheffield Children's NHS Foundation Trust; Ruth Logan, Sheffield Teaching Hospitals NHS Foundation Trust; Deborah Turner, South Devon Healthcare NHS Trust; Dianne Plews, South Tees Hospitals NHS Trust; Juliah Jonasi, Southend University Hospital NHS Foundation Trust; Ruth Pettengell, St George's Healthcare NHS Trust; Kamal Khoobarry, Surrey and Sussex Healthcare NHS Trust; Angela Watts, The Dudley Group of Hospitals NHS Foundation Trust; Louise Soanes, The Royal Marsden NHS Foundation Trust; Claudette Jones, The Royal Orthopaedic Hospital NHS Trust; Michael Jenkinson, The Walton Centre for Neurology and Neurosurgery NHS Trust; Nicky Pettitt, University Hospital Birmingham NHS Foundation Trust; Vijay Agarwal, University Hospital Birmingham NHS Foundation Trust; Beth Harrison, University Hospitals Coventry and Warwickshire NHS Trust; Fiona Miall, University Hospitals of Leicester NHS Trust; Gail Wiley, University Hospitals of Morecambe Bay NHS Trust; Lynda Wagstaff, Walsall Hospitals NHS Trust; Fiona Smith, West Hertfordshire Hospitals NHS Trust; Sarah Janes, Western Sussex NHS Trust; Serena Hillman, Weston Area Health NHS Trust; Christopher Zaborowski, Yeovil District Hospital NHS Foundation Trust. Data for this study is based on information collected and quality assured by the PHE National Cancer Registration and Analysis Service. Access to the data was facilitated by the PHE 
Office for Data Release. The Quality of Life study described in this paper was carried out using the PedsQL, developed by Dr James W Varni.

Contributors RMT, LAF, JB, DPS, SM, RF, LH, FG, RR and JW were involved in developing the protocol. RMT coordinated the running of the study and was responsible for data acquisition. JB, JA-G, RMT, LAF, SM, SL, AM, RF, DPS, JW contributed to the analysis. All authors critically revised and approved the final manuscript.

Funding This paper presents independent research funded by the National Institute for Health Research (NIHR) under its Programme Grants for Applied Research Programme (Grant Reference Number RP-PG-1209-10013). The views expressed are those of the author(s) and not necessarily those of the NHS, the NIHR or the Department of Health. The BRIGHTLIGHT Team acknowledges the support of the NIHR, through the Cancer Research Network. LAF and LH are funded by Teenage Cancer Trust, DPS holds research grant funding from Teenage Cancer Trust, and RR was (in part) supported by the National Institute for Health Research (NIHR) Collaboration for Leadership in Applied Health Research and Care (CLAHRC) North Thames at Bart's Health NHS Trust. RMT is a National Institute for Health Research (NIHR) Senior Nurse Research Leader. The views expressed are those of the author(s) and not necessarily those of the NIHR or the Department of Health and Social Care. None of the funding bodies have been involved with study concept, design or decision to submit the manuscript. JA-G was subsidised by the Ramon \& Cajal programme operated by the Ministry of Economy and Business (RYC-201619353), and the European Social Fund.

Competing interests None declared.

Patient consent for publication Not required.

Ethics approval The study was approved by the Health Research Authority Confidentiality Advisory Group (reference ECC 8-05(d)/2011) and London Bloomsbury NHS Research Ethics Committee (reference L0/11/1718).

Provenance and peer review Not commissioned; externally peer reviewed.

Data availability statement Data are available upon reasonable request. Further details of the BRIGHTLIGHT programme of work are available through the study website (www.brightlightstudy.com). Data that are not held under licence with Public Health England or NHS Digital will be available from late 2020 when the primary analysis is complete. We welcome collaboration, for general data sharing enquiries please contact RMT (rtaylor13@nhs.net).

Supplemental material This content has been supplied by the author(s). It has not been vetted by BMJ Publishing Group Limited (BMJ) and may not have been peer-reviewed. Any opinions or recommendations discussed are solely those of the author(s) and are not endorsed by BMJ. BMJ disclaims all liability and responsibility arising from any reliance placed on the content. Where the content includes any translated material, BMJ does not warrant the accuracy and reliability of the translations (including but not limited to local regulations, clinical guidelines, terminology, drug names and drug dosages), and is not responsible for any error and/or omissions arising from translation and adaptation or otherwise.

Open access This is an open access article distributed in accordance with the Creative Commons Attribution Non Commercial (CC BY-NC 4.0) license, which permits others to distribute, remix, adapt, build upon this work non-commercially, and license their derivative works on different terms, provided the original work is properly cited, appropriate credit is given, any changes made indicated, and the use is non-commercial. See: http://creativecommons.org/licenses/by-nc/4.0/.

\section{ORCID iDs}

Rachel M Taylor http://orcid.org/0000-0002-0853-0925

Javier Alvarez-Galvez http://orcid.org/0000-0001-9512-7853

Stephen Morris http://orcid.org/0000-0002-5828-3563

Jeremy Whelan http://orcid.org/0000-0001-6793-5722

\section{REFERENCES}

1 Barr RD. Adolescents, young adults, and cancer-the international challenge. Cancer 2011;117:2245-9.

2 Wang Y, Zhou S, Yang F, et al. Treatment-Related adverse events of PD-1 and PD-L1 inhibitors in clinical trials: a systematic review and meta-analysis. JAMA Oncol 2019;5:1008-19.

3 National Institute for Health and Care Excellence. Guidance on cancer services: improving outcomes in children and young people with cancer. London: NICE, 2005. https://www.nice.org.uk/guidance/ csg7/resources/improving-outcomes-in-children-and-young-peoplewith-cancer-update-773378893

4 Stark D, Bielack S, Brugieres L, et al. Teenagers and young adults with cancer in Europe: from national programmes to a European integrated coordinated project. Eur J Cancer Care 2016;25:419-27.

5 Barr RD. Planning a comprehensive program in adolescent and young adult oncology-a collision with reality. J Adolesc Young Adult Oncol 2016;5:303-9.

6 Fern L, Taylor RM, Whelan J, et al. 'The art of age appropriate care': using participatory research to describe young people's experience of cancer. Cancer Nursing 2013;36:E27-38.

7 Morgan S, Davies S, Palmer S, et al. Sex, drugs, and rock 'n' roll: caring for adolescents and young adults with cancer. J Clin Oncol 2010;28:4825-30.

8 Sironi G, Barr RD, Ferrari A. Models of care-there is more than one way to deliver. The Cancer Journal 2018;24:315-20.

9 Taylor RM, Fern L, Whelan J, Millington $\mathrm{H}$, et al. "Your place or mine?" Priorities for a specialist teenage and young adult (TYA) cancer unit: disparity between TYA and professional perceptions. $J$ Adolesc Young Adult Oncol 2011;1:145-51.

10 Rae CS, Pole JD, Gupta S, et al. Development of system performance indicators for adolescent and young adult cancer care and control in Canada. Value Health 2020;23:74-88.

11 Whiteson M. The Teenage Cancer Trust--advocating a model for teenage cancer services. Eur J Cancer 2003;39:2688-93.

12 Osborn M, Johnson R, Thompson K, et al. Models of care for adolescent and young adult cancer programs. Pediatr Blood Cancer 2019;66:e27991.

13 Smith AW, Bellizzi KM, Keegan THM, et al. Health-related quality of life of adolescent and young adult patients with cancer in the United States: the adolescent and young adult health outcomes and patient experience study. J Clin Oncol 2013;31:2136-45

14 Department of Health. The NHS constitution: the NHS belongs to us all. London: $\mathrm{DoH}, 2015$.

15 Henderson R. What is the NHS? An introduction to the NHS 2020.

16 O'Hara C, Khan M, McCabe M, et al. Notifications of teenagers and young adults with cancer to a principal treatment centre 2009-2010. National cancer intelligence network, 2013. Available: http://www. ncin.org.uk/view?rid=2124

17 Lea S, Taylor RM, Martins A, et al. Conceptualising age-appropriate care for teenagers and young adults with cancer: a qualitative mixed methods study. Adolescent Health Medicine and Therapeutics 2018;9:149-66.

18 Taylor RM, Feltbower RG, Aslam N, et al. Modified international e-Delphi survey to define healthcare professional competencies for working with teenagers and young adults with cancer. BMJ Open 2016;6:e011361.

19 Gibson F, Fern L, Whelan J, et al. A scoping exercise of favourable characteristics of professionals working in teenage and young adult cancer care: 'thinking outside of the box'. Eur J Cancer Care 2012;21:330-9.

20 Taylor RM, Whelan JS, Gibson F, et al. Involving young people in BRIGHTLIGHT from study inception to secondary data analysis: insights from 10 years of user involvement. Res Involv Engagem 2018;4:50.

21 Taylor RM, Gibson F, Franck LS. A concept analysis of health-related quality of life in young people with chronic illness. $J$ Clin Nurs 2008; 17:1823-33.

22 Quinn GP, Gonçalves V, Sehovic I, et al. Quality of life in adolescent and young adult cancer patients: a systematic review of the literature. Patient Relat Outcome Meas 2015;6:19-51.

23 Husson O, Zebrack BJ, Block R, et al. Health-Related quality of life in adolescent and young adult patients with cancer: a longitudinal study. J Clin Oncol 2017;35:652-9.

24 Taylor RM, Fern LA, Solanki A, et al. Development and validation of the BRIGHTLIGHT survey, a patient-reported experience measure for young people with cancer. Health Qual Life Outcomes 2015;13:107.

25 Taylor RM, Fern LA, Barber J, et al. Description of the BRIGHTLIGHT cohort: the evaluation of teenage and young adult cancer services in England. BMJ Open 2019:9:e027797.

26 Taylor RM, Aslam N, Lea S, et al. Optimizing a retention strategy with young people for BRIGHTLIGHT, a longitudinal cohort study examining the value of specialist cancer care for young people. $J$ Adolesc Young Adult Oncol 2017;6:459-69.

27 Taylor RM, Mohain J, Gibson F, et al. Novel participatory methods of involving patients in research: naming and branding a longitudinal cohort study, BRIGHTLIGHT. BMC Med Res Methodol 2015;15:20.

28 Taylor RM, Solanki A, Aslam N, et al. A participatory study of teenagers and young adults views on access and participation in cancer research. Eur J Oncol Nurs 2016;20:156-64. 
29 Martins A, Taylor RM, Lobel B, et al. Sex, body image, and relationships: a BRIGHTLIGHT workshop on information and support needs of adolescents and young adults. J Adolesc Young Adult Oncol 2018;7:572-8.

30 Taylor RM, Lobel B, Thompson K, et al. BRIGHTLIGHT researchers as 'dramaturgs': creating there is a Light from complex research data. Res Involv Engagem 2020;6:48.

31 Kenten C, Martins A, Fern LA, et al. Qualitative study to understand the barriers to recruiting young people with cancer to BRIGHTLIGHT: a national cohort study in England. BMJ Open 2017;7:e01829.

32 Varni JW, Burwinkle TM, Seid M, et al. The PedsQL 4.0 as a pediatric population health measure: feasibility, reliability, and validity. Ambu Pediatr 2003;3:329-41.

33 Varni JW, Burwinkle TM, Katz ER, et al. The PedsQL in pediatric cancer: reliability and validity of the pediatric quality of life inventory generic core scales, multidimensional fatigue scale, and cancer module. Cancer 2002;94:2090-106.

34 Fredericks EM, Magee JC, Opipari-Arrigan L, et al. Adherence and health-related quality of life in adolescent liver transplant recipients. Pediatr Transplant 2008;12:289-99.

35 King M, Jones L, Richardson A, et al. The relationship between patients' experiences of continuity of cancer care and health outcomes: a mixed methods study. Br J Cancer 2008;98:529-36.
36 Hsieh FY, Bloch DA, Larsen MD. A simple method of sample size calculation for linear and logistic regression. Stat Med 1998;17:1623-34.

37 Varni JW, Limbers CA. The PedsQL 4.0 generic core scales young adult version: feasibility, reliability and validity in a university student population. J Health Psychol 2009;14:611-22.

38 Department for Communities and Local Government. The English indices of deprivation 2015 statistical release. England: Department for Communities and Local Government, 2015.

39 Henderson R, Diggle P, Dobson A. Joint modelling of longitudinal measurements and event time data. Biostatistics 2000;1:465-80.

40 Fulop NJ, Ramsay AIG, Perry C, et al. Explaining outcomes in major system change: a qualitative study of implementing centralised acute stroke services in two large metropolitan regions in England. Implement Sci 2016;11:80.

41 Vindrola-Padros C, Taylor RM, Lea S, et al. Mapping adolescent cancer services: how do young people, their families, and staff describe specialized cancer care in England? Cancer Nurs 2016;39:358-66.

42 Kreuter F, Presser S, Tourangeau R. Social desirability bias in cati, IVR, and web surveys: the effects of mode and question sensitivity. Public Opin Q 2008;72:847-65. 